\title{
Lidil
}

Revue de linguistique et de didactique des langues

$50 \mid 2014$

Variation stylistique et diversité des contextes de socialisation

\section{Choix stylistiques plurilingues, catégorisation et construction de sens : étude exploratoire dans une école professionnelle de Vénétie (Italie)}

Multilingual Stylistic Choices, Categorization and Construction of Meaning: A

Pilot Study in a Professional School in Veneto (Italy)

\section{Anna Ghimenton et Letizia Volpin}

\section{OpenEdition}

\section{Journals}

Édition électronique

URL : http://journals.openedition.org/lidil/3569

DOI : 10.4000/lidil.3569

ISSN : 1960-6052

\section{Éditeur}

UGA Éditions/Université Grenoble Alpes

\section{Édition imprimée}

Date de publication : 15 décembre 2014

Pagination : 63-79

ISBN : 978-2-84310-287-5

ISSN : $1146-6480$

\section{Référence électronique}

Anna Ghimenton et Letizia Volpin, « Choix stylistiques plurilingues, catégorisation et construction de sens : étude exploratoire dans une école professionnelle de Vénétie (Italie)», Lidil [En ligne], $50 \mid 2014$ mis en ligne le 15 juin 2016, consulté le 30 avril 2019. URL : http://journals.openedition.org/lidil/3569 ; DOI : 10.4000/lidil.3569 


\title{
Choix stylistiques plurilingues, catégorisation et construction de sens : étude exploratoire dans une école professionnelle de Vénétie (Italie)
}

\author{
Anna Ghimenton* et Letizia Volpin**
}

\begin{abstract}
RÉSUMÉ
Cette contribution présente une étude exploratoire menée dans le cadre scolaire en Vénétie (nord-est de l'Italie). Nous examinons les pratiques langagières de 68 élèves (adolescents) inscrit-e-s dans une école professionnelle de Noale, une ville de 15000 habitants près de Venise. Leurs usages langagiers se situent sur un continuum allant d'un pôle dialectal à un pôle italien. Nous proposons une analyse à partir de données recueillies dans le cadre de 150 heures d'observation participante. L'objectif de cette recherche est de mieux comprendre la façon dont les productions plurilingues en classe contribuent à la variation diaphasique observée dans les pratiques interactionnelles d'un groupe d'adolescents. Nous explorons ainsi les catégorisations qui contribuent à la construction de sens chez les adolescents de Vénétie, observés dans un contexte communicationnel partagé (activités de classe).
\end{abstract}

\begin{abstract}
This paper presents a pilot study conducted in a school in Veneto (northeastern Italy). We examine the language practices of 68 learners (adolescents) enrolled in a technical school in Noale, a town with 15000 inhabitants, close to Venice. Their language usages are situated along an Italian-dialect continuum. The data were collected in our 150 hours of participant observation. In the analyses, we examine the ways in which the adolescents exploit their multilingualism for stylistic purposes within the school context. Our analyses provide a different approach to style, as we do not consider social stratification (the adolescents come
\end{abstract}

* Université Sorbonne Nouvelle - Paris 3, CLESTHIA.

** Université de Neuchâtel. 
from the same social background). Rather, we explore the categorisations and the processes of social meaning construction that emerge in a shared communicative context (classroom activities).

What people think cannot be denied ${ }^{1}$.

(Proverbe basakata)

\section{Introduction}

Un regard attentif sur les différentes études centrées sur le style permet de constater une variation importante dans les approches théoriques et méthodologiques. Cette variation est particulièrement frappante si on considère la richesse des contextes sociolinguistiques explorés ainsi que la diversité de questions de recherche posées concernant le style. L'étude de la variation diaphasique ${ }^{2}$ revêt donc un champ protéiforme, et - étant relativement nouvelle en particulier dans un contexte plurilingue - contribue à une meilleure connaissance des liens sociaux s'établissant entre style et pratiques langagières des jeunes. À notre connaissance, dans le domaine italoroman, peu d'études à caractère ethnographique ont été menées dans l'espace scolaire auprès d'adolescents. Le but de cette recherche exploratoire est de mieux comprendre la façon dont un groupe de jeunes adolescent-e-s mettent leur plurilinguisme à contribution de la variation stylistique dans leurs pratiques interactionnelles au sein de la sphère scolaire. Ces pratiques se caractérisent par l'usage conjoint de l'italien et du dialecte vénitien dans les conversations entre pairs mais aussi dans les tours de paroles que les élèves adressent à leurs professeurs. À partir d'un corpus d'annotations, nous nous interrogeons sur les «mouvements stylistiques» qui traversent les espaces discursifs observés lors d'une période d'observation participante effectuée dans l'école professionnelle en essayant d'en saisir la complexité. Ainsi, les caractéristiques sociolinguistiques du contact des langues observées dans notre terrain de recherche nous ont conduites à rapprocher la théorie de l'audience design présentée

1. Traduction : «Ce que pensent les gens ne peut être nié.»

2. Tout en étant conscientes de la complexité de la dimension diaphasique, ne se réduisant pas à la seule composante «style», dans le cadre de notre présentation, nous focalisons notre attention sur cette composante et utilisons les termes diaphasique et stylistique de manière interchangeable. 
par Bell (1984) à la théorie de l'identité sociale (Social identity theory) avancée par Tajfel (1978) issue de la psychologie sociale. La revue des travaux présentée dans la section suivante situe la dimension du style dans ce rapprochement disciplinaire dans le contexte italoroman.

\section{De la théorie de l'identité sociale à l'accommodation : un rapprochement des niveaux intra- et inter-individuels?}

Les travaux laboviens (entre autres, Labov, 1972) marquent les débuts de la sociolinguistique variationniste et des approches quantitatives, fondées sur l'examen de la variation stylistique en fonction de facteurs sociaux (voir Schilling-Estes, 2002, pour une vue synoptique des travaux en question). Il s'agit d'une approche «statistique-corrélationnelle» (Mendoza-Denton, 2010, p. 186) permettant de relier les phénomènes de variation stylistique aux caractéristiques sociales du locuteur. En effet, dans un souci de quantification des données, les éléments interactionnels sous-tendant la production langagière passent au deuxième plan car les variables observées seraient mises en corrélation avec l'appartenance sociale des locuteurs. À la même époque, Hymes (1974) introduit dans le paysage sociolinguistique l'ethnographie de la communication où une approche plus qualitative de l'interaction est envisagée. Quelle que soit l'approche adoptée, les études sur la variation stylistique situées dans les espaces idéologiquement monolingues d'Europe ou d'Amérique du Nord tendent à privilégier la perspective intra-locuteur dans la prise en compte de la dimension diaphasique. Une nouvelle lignée d'études marque pourtant le mouvement progressif vers un rapprochement entre l'individuel et le social (Buson, 2008; Eckert, 2000; Jaspers, 2011 ; Podesva, 2008). Dans ces travaux - tous centrés, à l'exception de Jaspers (2011), sur le contexte monolingue - on souligne l'importance de ramener l'intra-individuel (niveau privilégié pour l'étude du style) vers l'inter-individuel, restituant ainsi la réalité sociale aux pratiques langagières individuelles. Ce rapprochement devient en effet une démarche évidente lorsque le style est étudié dans des aires plurilingues. Dans leurs nombreux travaux menés dans les aires créolophones guyanaises, Migge et Léglise (2011, p. 225) remarquent que les dimensions inter- et intra-individuelles sont imbriquées puisque les individus puisent dans des ressources linguistiques et sociales pour ensuite construire des identités individuelles et collectives : 
Essentially, in accordance with their goals, speakers draw in selective ways on the locally available linguistic and social resources, including ethnically neutral forms, to construct unique individual and group identities. Linguistically, this leads to a kind of reorganization and linguistic instability that over time develops into a stable variation and codeswitching and gives rise to the emergence of new styles.

Migge et Léglise exposent des mouvements qui fluctuent entre la diversification et la stabilisation des formes linguistiques. Ces mouvements - apparemment contradictoires - sous-tendent les processus de construction de sens à l'échelle individuelle et collective où l'on voit émerger des variétés et des styles nouveaux (Migge \& Léglise, 2011, 2013). Ainsi, la dissociation des dimensions intra- et inter-locuteur aboutirait à une analyse du style morcelée voire incomplète, en particulier si l'on s'y intéresse dans un contexte de contact des langues.

C'est dans une telle dynamique, entre les langues en contact d'une part et l'individu et le groupe d'autre part, que la théorie de l'identité sociale de Tajfel (1978) offre des outils d'analyse opérationnels ${ }^{3}$, particulièrement utiles si on s'intéresse au plurilinguisme et au style. D'après cette théorie, chaque individu s'identifie avec un ensemble de personae, dont certaines ont un caractère plutôt idiosyncrasique (et donc spécifiques à l'individu) tandis que d'autres relèvent des identités collectives et partagées. Meyerhoff (2011) résume les principes de la théorie sociale de l'identité en précisant comment celle-ci rend compte de la variation des pratiques. Par exemple, lorsqu'une identité individuelle est saillante (en fonction de paramètres variables selon les locuteurs et le contexte de production), les pratiques seront contraintes par les aspects idiosyncrasiques caractérisant le locuteur. Ainsi, au cours d'une telle interaction, la théorie de Tajfel prévoit une plus grande variabilité des usages. En revanche, lorsque l'identité du groupe est plus saillante, alors les pratiques langagières des locuteurs seront davantage homogènes dans un souci de se conformer à, ou de prendre de la distance par rapport à des normes partagées ou non. Une identité n'est donc pas saillante en soi : elle est le fruit d'une négociation, voire d'une coconstruction, qui se fait au cours de l'interaction (voir Eckert, 2008) au sein de laquelle certains traits deviennent plus saillants que d'autres pour les interlocuteurs participant à l'échange. Au cours de l'activité

3. Pour une excellente présentation synthétique de la théorie de Tajfel (1978), voir Meyerhoff (2011). 
langagière, le locuteur viserait le regard favorable ou admirateur de son interlocuteur (cf. Sedikides \& Strube, 1997; Tajfel, 1978), ou bien chercherait à s'en démarquer. Quelle que soit l'orientation du locuteur engagé dans l'interaction, son activité langagière marquerait ainsi simultanément un positionnement (qu'il soit physique ou symbolique) par rapport à l'autre qui ferait des deux parties un sujet catégorisé et catégorisant : en empruntant les termes de Wald (1990), «[t]out acte de parole est trace de catégorisation sociale et tout effet de discours passe par son interprétation» (p. 6). Il est d'ailleurs possible d'entrevoir des similarités entre cette théorie et la notion de cadre participatif de Goffman (1981), selon laquelle l'individu peut assumer un ensemble de positions vis-à-vis de son discours et celle de audience design de Bell (1984), selon laquelle la présence de l'auditoire exerce une influence sur la production langagière de l'individu. Cependant, à notre avis, la théorie de Tajfel rend mieux compte de la dynamique entre l'individu et le groupe, et s'avère donc une théorie particulièrement prometteuse pour l'analyse de nos données recueillies en Vénétie.

\section{Contacts de langues dans l'aire italoromane : un bref aperçu sociolinguistique des répertoires et des usages}

Longtemps caractérisée par la présence de langues en contact, l'aire italoromane reste encore aujourd'hui un espace géolinguistique plurilingue. Dans le domaine italoroman ${ }^{4}$, l'italien est en contact avec les dialectes régionaux, qui sont des langues locales issues directement du latin, tout comme le toscan à partir duquel l'italien «langue nationale» s'est ensuite développé. De ce contact «langue nationale-langues régionales» naissent des variations régionales de l'italien (Cammarota, 1997). C'est d'ailleurs pour cette raison qu'on ne peut pas parler d'italien standard per se, pour le moins en ce qui concerne sa réalisation orale car il varie selon les régions (Berruto, 1993). Ainsi, dans le cadre de cet article, lorsque nous nous référons à «italien» tout court, il s'agit en réalité de l'italien dans ses réalisations régionales de Vénétie. Par ailleurs, il est important de préciser que les dialectes ne sont pas des

4. Nous n'incluons pas, dans le cadre de cette présentation, les langues issues de l'immigration, bien qu'elles contribuent au plurilinguisme de l'espace italoroman. Nous présentons un survol historique des caractéristiques sociolinguistiques de l'aire italoromane. 
dérivés ou des simplifications de l'italien (Berruto, 2005). En effet, chaque dialecte a un système linguistique qui lui est propre bien qu'il soit génétiquement apparenté avec l'italien. Les études sociolinguistiques du contact menées dans cette aire montrent que les pratiques langagières varient au long d'un continuum allant d'un pôle dialectal vers un pôle italien (Berruto, 1993). Par exemple, D'Achille et Giovanardi (1995) notent que même si les interactions se déroulent en italien, la présence de traits dialectaux dans les pratiques est remarquable. Ce va-et-vient entre langues en contact représente un moyen que se donnent les locuteurs pour élargir leur éventail stylistique. En effet, là où le locuteur monolingue mobilise les ressources stylistiques de sa langue, le locuteur plurilingue puise dans son répertoire langagier pour mobiliser les ressources stylistiques de chacune de ses langues. Il peut également opter pour l'utilisation conjointe de ces dernières pour atteindre ces mêmes effets communicationnels (Gumperz, 1982).

Dans une perspective ethnographique, ce travail exploratoire se donne comme objectif d'examiner la variation des pratiques stylistiques plurilingues d'adolescent-e-s dans une situation de classe. Nous nous interrogeons sur le rôle des paramètres de la situation de communication (locuteur, interlocuteur, contexte) dans la variation stylistique des adolescent-e-s et nous essayons de comprendre comment cette variation illustre leur souplesse stylistique.

\section{Méthodologie : les sujets et les données}

Cette recherche a eu lieu dans le cadre d'une période d'observation participante de 150 heures dans une école professionnelle ${ }^{5}$ en Italie et s'est déroulée pendant l'année scolaire 2012-2013. L'école est située à Noale en Vénétie, une des régions nord-orientales de l'Italie. C'est une commune de 15000 habitants située à une vingtaine de kilomètres de Venise. Avant de commencer les observations, nous avons contacté les professionnels de l'école et nous leurs avons expliqué les grandes lignes des objectifs de notre enquête. Une fois obtenu l'accord de la directrice de l'école, les observations ont démarré aussitôt. L'observatrice s'est assise au fond de la classe parmi les élèves du dernier rang.

5. L'école professionnelle en Italie correspond au lycée professionnel en France dont le but est de transmettre aux élèves des connaissances et des savoir-faire indispensables pour leur entrée dans le monde du travail. 
L'âge des élèves dont les pratiques ont été observées varie entre 16 et 21 ans. Rappelons que la fourchette d'âge est aussi étendue car les écoles professionnelles accueillent souvent des élèves en situation d'échec scolaire. Le but de ces écoles est d'aider les élèves dans leur insertion dans le monde du travail. C'est le cas, en particulier, de l'école qui constitue le terrain de cette enquête qui propose plusieurs projets d'insertion professionnelle. À titre d'exemple, nous en citons un qui a été développé lors de notre présence : les élèves devaient réfléchir à la création d'une coopérative sociale. Le nom de la coopérative, Source of Help (angl. «Source d'aide») a été choisi par les élèves. Il semble révéler que l'école est vue par les élèves comme une aide à l'insertion professionnelle.

Trois classes ont été observées : des apprentis mécaniciens, des apprentis électriciens et des apprentis secrétaires ${ }^{6}$. Les groupes des mécaniciens et des électriciens sont constitués exclusivement de garçons alors que la cohorte de secrétaires, à l'exception de deux garçons d'origine étrangère, est constituée de filles. La plupart des élèves sont d'origine italienne. Les élèves d'origine étrangère sont en minorité et proviennent de différents pays : Albanie, Chine, Roumanie, Maroc et Sénégal ${ }^{7}$.

L'approche méthodologique de ce travail exploratoire est donc à caractère ethnographique ${ }^{8}$. S'agissant des phases initiales d'une recherche de terrain, les considérations présentées ici sont issues de la réélaboration d'un nombre important de notes prises pendant les observations de classe ou à postériori une fois terminée la journée d'école.

6. Pour des raisons de simplicité, dorénavant nous désignons les apprentis mécaniciens, les apprentis électriciens et les apprentis secrétaires respectivement «mécaniciens », «électriciens » et «secrétaires».

7. Concernant les usages plurilingues des élèves étrangers, nous n'avons pas repéré des usages particuliers de leurs langues premières, sans doute parce que d'une part ils ne proviennent pas de la même aire géographique et ne partagent pas les mêmes langues et, d'autre part, les interactions de classe observées n'offraient pas d'occasions pour s'exprimer dans des langues autres que l'italien et le dialecte.

8. Pour une introduction à l'approche ethnographique adoptée dans des travaux centrés sur le plurilinguisme, voir Heller (2009). 


\section{Analyses des pratiques langagières}

La variation diaphasique dans le langage des jeunes a été explorée par plusieurs sociolinguistes travaillant dans des contextes géolinguistiques divers, par exemple dans le contexte roman (Auzanneau, LeclèreMessebel \& Juillard, 2012; Buson, 2008; Cerruti, 2003, 2004 ; Depau, 2011 ; Fusco, 2007; Lambert, 2009) et dans le contexte anglo-saxon (Eckert, 1989, 2008; Schilling-Estes, 2002; Snell, 2010). En continuité avec ces travaux, nous explorons la façon dont le contexte influence les choix codiques et stylistiques des jeunes. La notion de contexte est centrale pour les études portant sur la variabilité langagière (entre autres, Auer, 1992; Ervin-Tripp, 1996; Duranti \& Goodwin, 1992 ; Kerbrat-Orecchioni, 2005, 2006; Mondada, 2006) et demeure un objet de réflexion et de débat. Tous ces travaux portent - à travers des démarches différentes - sur une analyse du langage dans une perspective discursive (lato sensu), caractérisant aussi notre approche d'étude. Nous tenons compte des dimensions interactionnelle et relationnelle, que nous considérons comme inextricables lors d'une prise en charge de l'espace physique et symbolique caractérisant l'échange verbal. Autrement dit, nous portons notre attention sur les contextes d'interaction en examinant les relations qu'entretiennent les interlocuteurs et les rapports qui se forgent au cours de l'activité langagière.

\subsection{Pratiques du groupe d'apprentis électriciens et d'apprentis mécaniciens : flottements entre déviance et connivence}

Comme nous l'avons précisé plus haut (voir section Méthodologie : les sujets et les données), le groupe d'électriciens et de mécaniciens est constitué exclusivement de garçons. Dans ce groupe, la fonction expressive du dialecte est très productive et varie selon les contextes communicatifs. En observant les diverses pratiques langagières, nous avons repéré certains cas où le dialecte sert en quelque sorte à exprimer une provocation ou une bravade : le locuteur qui produit la séquence dialectale se positionne en situation de force par rapport à son interlocuteur, en particulier si celui-ci représente le système hiérarchisé échafaudant la structure scolaire. Nous reproduisons trois exemples illustrant ce type de productions dialectales réalisées par les élèves en direction de leurs enseignants :

(1) «Prof, vien qua! »

Traduction : «Prof, viens ici!» 
(2) «Quanti schèi me dètu?»

Traduction : «Combien d'argent tu me donnes?»

(3) «Sò stùfo, go sòno!»

Traduction : «Je suis fatigué, j’ai sommeil !»

(4) «Ma che casso me firmito! »

Traduction : «Mais qu'est-ce que tu me signes, bordel!»

Remarquons que les élèves ne vouvoient pas les professeurs, bien que ces derniers l'exigent. Cependant, les élèves connaissent les règles présidant aux usages ou encore les formes plus soutenues, car ils vouvoient la directrice et l'observatrice. Ceci suggère que l'absence de vouvoiement serait un choix délibéré des élèves. Le registre de ces quatre productions est aux antipodes de celui sous-tendant les formes langagières attendues dans l'échange entre élève et professeur. Précisons que l'écart observé entre l'usage attendu et celui effectif ne se manifeste pas par l'usage du dialecte (les professeurs l'utilisent aussi) mais par les registres choisis puisés dans leurs répertoires dialectaux.

À l'instar des observations de Ursini (2005, p. 322), cet usage du dialecte est le symptôme d'une exploration stylistique-énonciative qui marque l'éloignement vis-à-vis d'une norme standard, monolithique, représentée par le professeur. Dans les quatre premiers exemples, cette exploration stylistique-énonciative se manifesterait de différentes manières. Premièrement, les exemples (1) et (2) illustrent un dépassement, voire l'adoption d'un statut dominant autorisant l'élève à donner des ordres au professeur. Deuxièmement, l'élève qui produit l'exemple (3) déclare avoir sommeil et être fatigué, signifiant donc un refus vis-àvis de l'accomplissement de la tâche attendue. L'exemple (4) illustre que l'usage du dialecte peut être accompagné par un registre vulgaire, notamment par le recours de l'injure casso 'bite'. Ce type d'usage a été également repéré dans les interactions entre jeunes par Sobrero $\&$ Miglietta (2003) dans le sud de l'Italie où les pratiques dialectales observées se caractérisent par la présence d'injures (cf. exemple 4), d'exclamations (cf. exemples 1 à 4) ou de commentaires par rapport à un état d'esprit (cf. exemple 3 ) ou à une conduite de son propre interlocuteur (cf. exemple 4).

Cependant, ce type de décalage par rapport au style attendu dans l'interlocution en situation formelle ne se produit pas systématiquement. Avec un professeur en particulier, les élèves des deux groupes ont une relation de confiance et d'estime mutuelle. Ce n'est pas étonnant de voir un changement de registre chez les élèves dans les interactions 
avec ce professeur. En effet, ils utilisent les formes de politesse (Lei), même si parfois il y a des glissements vers les formes d'adresse plus familières (prof au lieu de professore). La dimension relationnelle entre les interactants influencerait donc leurs choix codiques et stylistiques qui tendent - dans le cadre des interactions avec ce professeur - vers un pôle plus formel :

(5) «Arrivederci prof, buon fine settimana.»

Traduction : «Au revoir prof, bonne fin de semaine.»

(6) «Lei mi fa amare queste cose.»

Traduction : «Vous me faites aimer ces choses-là. »

Ces deux énoncés produits en italien (5) et (6) divergent nettement des énoncés (1) - (4) qui - comme on l'a vu - sont en décalage par rapport aux productions attendues au sein du cadre institutionnel et à la situation d'apprentissage ${ }^{9}$. Dans les deux exemples en question, les élèves utilisent le langage de politesse (ex. vouvoiement, formules de politesse et de salutation). L'énoncé (5) a été produit par un élève qui, une fois le cours terminé, donne congé au professeur en lui souhaitant une bonne fin de semaine. L'exemple (6) fournit un autre exemple de production d'un élève vers ce professeur. Le jeune exprime sa reconnaissance au professeur qui lui fait aimer queste cose 'ces choses-là', des termes génériques pour désigner les apprentissages scolaires. Il semblerait que les productions langagières des élèves dans le contexte de communication avec ce professeur montrent une convergence avec le style attendu pour une séquence formelle, mais aussi une convergence avec les objectifs pédagogiques de la sphère scolaire, à savoir la transmission réussie des connaissances.

\subsection{Pratiques langagières du groupe des secrétaires : plurilinguisme et catégorisations}

Le groupe des secrétaires est composé principalement de filles, à l'exception de deux garçons d'origine étrangère. Ces deux garçons n'ont que très rarement pris la parole dans les échanges auxquels nous avons assisté, ils étaient en retrait par rapport aux filles du groupe et aux professeurs. Parallèlement à ce que nous avons observé chez le groupe

9. Bien que les interactions avec ce professeur puissent se dérouler en dialecte, nous avons remarqué ici que les élèves n'ont pas recours au registre vulgaire de cette langue. 
d'élèves précédent, celui des secrétaires n'emploie pas les formes de politesse lorsqu'elles s'adressent au professeur donnant cours. Ces élèves - tout comme les mécaniciens et les électriciens - les utilisent toutefois en présence de la directrice ou de l'observatrice, bien que le registre que nous avons pu observer tende vers le pôle informel. Nous reportons deux exemples d'usage d'un registre informel de l'italien produit par deux filles en réponse à des questions posées par la professeure :

(7) «Ma che cazzo ne so?»

Traduction : «Mais qu'est-ce que j'en sais, bordel?»

(8) «Non capisco un cazzo.»

Traduction : «J'en sais foutre rien.»

Dans les exemples (7) et (8), les filles utilisent un registre informel de l'italien pour s'adresser au professeur. Remarquons ici qu'elles utilisent la version italienne de l'injure que nous avons relevée chez les garçons : ita. cazzo > dial. casso 'bite'. En revanche, dans le cas repéré ci-après, la locutrice réalise une alternance codique lorsqu'elle s'adresse à une camarade commençant par un fragment en dialecte jusqu'à la production de l'injure casso, puis elle passe à l'italien pour compléter son énoncé. Cette alternance pourrait contribuer à la mise en exergue de ses choix codiques et stylistiques en renforçant la fonction illocutoire sous-tendant son interrogation. Les parties en italique ont été réalisées en dialecte :

(9) «Te si fora, che casso stai dicendo? »

Traduction : «T'es cinglée, qu'est-ce que tu racontes, bordel?»

Globalement, les choix codiques des secrétaires se distinguent de ceux repérés chez les mécaniciens et les électriciens. Dans le groupe des secrétaires, l'italien est le choix codique de préférence dans les énoncés adressés aux professeurs. Toutefois, leurs pratiques sont souvent mélangées et on voit dialecte et italien entremêlés dans leurs énoncés. Contrairement à ce qui a été remarqué chez les garçons (cf. section précédente), les productions stylistiques des élèves de ce groupe sembleraient être moins variées, car le registre choisi dans les deux langues est - pour la plupart des cas - informel, qu'il s'agisse de conversations avec le professeur ou avec les pairs, comme il apparait dans les exemples (7) à (9).

Un dernier point mérite d'être précisé concernant les usages dialectaux du groupe en question. Parallèlement à ce qu'énonce Eckert (2008), 
nous observons que le dialecte a une position instable et fluctuante, comme si le contexte de production déterminait le sens social véhiculé par son usage ainsi que son statut immédiatement saisissable par les locuteurs partageant ce même contexte. Prenons les deux exemples suivants :

(10) «No sta far el figo.»

Traduction : «Ne te la pète pas.»

(11) «Boara»

Traduction : «Plouque»

Dans l'exemple (10), une des filles s'adresse - en dialecte - à un camarade qui est interrogé par la professeure. Le contexte formel de l'interrogation orale semble créer une tension dans la classe, une tension qui est rapidement brisée par l'expression dialectale familière. Puisque cette expression comporte un écart par rapport au style attendu, nous pouvons émettre l'hypothèse qu'elle freine toute convergence stylistique potentielle de la part de l'élève interrogé vers le langage appartenant à la sphère scolaire. Ici le dialecte serait, donc, le moyen linguistique et stylistique pour empêcher une telle convergence avec le langage associé à l'institution et peut-être une désolidarisation avec les caractéristiques du groupe.

Dans l'exemple (11), nous changeons de contexte car il s'agit d'un énoncé dialectal produit vers une élève qui, principalement dialectophone, rencontre des difficultés à s'exprimer en italien. Ici, c'est l'usage du dialecte - en tant que choix codique - qui fait l'objet d'une catégorisation de son utilisatrice, considérée comme un individu rustre et peu cultivé. En effet, boara, qui signifie littéralement «celle qui travaille dans l'élevage de vaches », appartient à un registre informel avec une connotation péjorative. Ainsi, il y aurait une convergence entre style choisi (choix codique) et catégorisation sociale, car l'usage dialectal devient à la fois l'objet et le moyen d'une telle catégorisation.

Dans l'état actuel de ce travail empirique, nous ne disposons pas de suffisamment de données pour mieux saisir les indices pragmatiques sous-tendant de telles catégorisations. Retenons tout de même que le dialecte manifeste une plurifonctionnalité stylistique, car son usage semble être plus «sensible» que celui de l'italien aux caractéristiques idiosyncrasiques de l'individu et à celles sous-tendant une identité collective. 


\subsection{Continuité stylistique des conduites verbales et non verbales?}

Dans les observations effectuées, plusieurs points de convergence ont été repérés entre le groupe des apprentis mécaniciens/électriciens et des apprentis secrétaires, en particulier lorsqu'on considère les conduites non verbales. En effet, nous avons remarqué une gestion de l'espace similaire. Les élèves ont une conduite décontractée, se déplacent dans la salle quand ils le souhaitent et ils ne lèvent pas la main quand ils souhaitent prendre la parole. En outre, le mobilier de la classe (tables et chaises) est utilisé pour poser leurs sacs ou leurs pieds : la plupart des élèves ne prend pas de notes lorsque le professeur expose son cours. Chez les «filles secrétaires», des activités stéréotypées ont été observées, telles que le maquillage. Ce rapport physique avec l'espace scolaire semble être tout à fait cohérent avec les pratiques langagières et stylistiques des élèves. Pensons par exemple aux usages familiers et provocateurs du dialecte en situation de classe. Au-delà des contrastes stylistiques déclenchés par l'usage du dialecte, il apparait que les élèves - à travers leurs mouvements dans la salle - s'approprient l'espace scolaire de manière physique/corporelle, là où la hiérarchisation des relations interpersonnelles (par exemple, le rapport élèves professeurs) ne leur permet pas de dominer l'espace discursif scolaire. Y a-t-il donc un continuum entre l'espace physique scolaire et les soubassements stylistiques de l'espace discursif des élèves? Pour que cette question trouve des réponses satisfaisantes, il est nécessaire de documenter cette continuité entre conduites non verbales et usages stylistiques des élèves à travers une étude ethnographique axée sur la multimodalité des échanges dans la classe. À notre avis, il s'agit d'une voie de recherche sur les styles qui pourrait apporter une dimension cognitive originale à l'analyse de ces données langagières.

\section{Conclusions}

Cette étude exploratoire a permis d'avoir un premier regard sur les pratiques langagières plurilingues et pluri-styles d'un groupe de jeunes adolescent-e-s d'une l'école professionnelle de Vénétie. Nous avons esquissé la manière dont les élèves - à travers leurs choix codiques et stylistiques - se positionnent par rapport à leurs interlocuteurs. D'abord, nos résultats suggèrent que le «potentiel stylistique» issu du contact entre le dialecte et l'italien ne serait pas exploité de manière égale chez les deux groupes observés. Les électriciens/mécaniciens mobilisent un répertoire plurilingue et stylistique plus ample que les 
secrétaires, car ils varient d'un registre vulgaire à un registre soutenu selon qu'ils souhaitent diverger ou converger avec leur interlocuteur. En revanche, les secrétaires s'aligneraient sur les choix stylistiques familiers de l'italien avec des glissements vers le dialecte. Ces glissements ne sont toutefois pas perçus de la même manière chez les deux groupes. Nous avons repéré que l'usage important du dialecte chez une élève a fait l'objet d'une catégorisation de cette locutrice comme étant une personne au faible répertoire langagier. De quelle manière ces catégorisations contribuent-elles à l'insécurité linguistique et à une réduction stylistique imposée par le regard «cloisonnant» d'autrui? Le matériel interactionnel à notre disposition ne nous permet pas d'apporter des éléments de réponse sur ce point. Ces premières observations nous amènent néanmoins à nous interroger sur la pertinence de la variable sexe dans l'exploration des pratiques stylistiques en milieu plurilingue. L'étude des différences filles/garçons mérite d'être approfondie grâce à la constitution d'un corpus audio-visuel de données interactionnelles.

De ce travail exploratoire, nous pouvons dégager deux lignes directrices pour de futures recherches ethnographiques. Premièrement, l'examen des (auto-)catégorisations des élèves selon leurs choix codiques et stylistiques permettrait d'observer la façon dont le procès de catégorisation du self et/ou d'autrui contribue à l'instauration tacite des «droits et obligations» codiques et stylistiques (cf. le principe des Rights and Obligations de Myers-Scotton \& Bolonyai, 2001) et de comprendre comment ils s'imprègnent du sens social négocié en contexte. Deuxièmement, une approche multimodale des pratiques dans l'espace scolaire pourrait apporter un éclairage sur les liens entre conduites verbales et non verbales qui, dans la dimension stylistique, doivent encore être explorés.

\section{RÉFÉRENCES BIBLIOGRAPHIQUES}

Auer, Peter. (1992). Introduction: John Gumperz' Approach to Contextualization. Dans P. Auer \& A. Di Luzio (dir.), The contextualization of language (p. 1-37). Amsterdam : Benjamins.

Auzanneau, Michelle, Leclère-Messebel, Malory \& Juillard, Caroline. (2012). Élaboration et théâtralisation de catégorisations sociolinguistiques en discours, dans une séance de formation continue. La catégorie «jeune» en question. Langage et société, 141(3), 47-69.

Bell, Allan. (1984). Language Style as Audience Design. Language in Society, 13, 145-204. 
Berruto, Gaetano. (1993). Varietà diamesiche, diastratiche, diafasiche. Dans A. Sobrero (dir.), L'italiano contemporaneo: la variazione e gli usi (volume II, p. 37-92). Rome-Bari : Laterza.

Berruto, Gaetano. (2005). Dialect/Standard Convergence, Mixing, and Models of Language Contact: The Case of Italy. Dans P. Auer, F. Hinskens \& P. Kerswill (dir.), Dialect Change. Convergence and Divergence in European Languages (p. 81-95). Cambridge : Cambridge University Press.

Buson, Laurence. (2008). La variation stylistique chez les enfants de 10/11 ans : une étude exploratoire en contexte français. Dans M. Abecassis et coll. (dir.), Le français parlé au XXI ${ }^{e}$ siècle (Normes et variations géographiques et sociales) (vol. 1, p. 203-222). Paris : L'Harmattan.

Cammarota, Marie-Ange. (1997). «Pygmalion» à l'italienne. Plurilinguismes, 13, 83-117.

Cerruti, Massimo. (2003). Il dialetto oggi nello spazio sociolinguistico urbano. Indagine in un quartiere di Torino. Rivista Italiana di dialettologia, 27, 33-88.

Cerruti, Massimo. (2004). Aspetti pragmatico-funzionali della commutazione di codice italiano dialetto: un'indagine a Torino. Vox Romanica, 63, 94-127.

D’Achille, Paolo \& Giovanardi, Claudio. (1995). Romanesco, neoromanesco o romanaccio? La lingua di Roma alle soglie del Duemila. Dans M. T. Romanello \& I. Tempesta. (dir.), Dialetti e lingue nazionali. Atti del XXVII Congresso della Società di Linguistica Italiana (Lecce, 2830 ottobre 1993) (p. 379-412). Rome : Bulzoni.

Dardano, Maurizio \& Trifone, Pietro. (2003). Grammatica italiana: con nozioni di linguistica ( $3^{\mathrm{e}}$ éd.). Bologne : Zanichelli.

Depau, Giovanni. (2011). Italien et sarde dans l'espace urbain de Cagliari : contact de langues et contexte d'interaction. CORELA. Disponible en ligne sur <http://corela.edel.univ-poitiers.fr/index.php?id=2179>.

Duranti, Alessandro \& Goodwin, Charles (dir.). (1992). Rethinking Context. Cambridge : Cambridge University Press.

ECKERT, Penelope. (1989). Jocks and Burnouts: Social Categories and Identity in the High School. New York : Teachers College Press.

ECKERT, Penelope. (2000). Linguistic Variation as Social Practice. Oxford, MA : Blackwell.

ECKERT, Penelope. (2008). Variation and the Indexical Field. Journal of Sociolinguistics, 12(4), 453-476.

Ervin-Tripp, Susan. (1996). Context in Language. Dans D. I. Slobin, J. Gerhardt, A. Kyratzis \& G. Jiansheng (dir.), Context in Language (p. 21-36). Mahwah, NJ : Lawrence Erlbaum Associates. 
Fusco, Fabiana. (2007). La lingua dei giovani in Italia: tratti e movimenti. Dans E. Neuland (dir.), Jugendsprachen. Mehrsprachig - kontrastiv interkulturell (p. 31-46). Francfort-sur-le-Main : Lang.

Giles, Howard. (1973). Accent Mobility: A Model and Some Data. Anthropological linguistics, 15, 87-105.

Goffman, Ervin. (1981). Forms of Talk. Philadelphie : University of Pennsylvania Press.

GumPERZ, John J. (1982). Discourse Strategies. New York : Cambridge University Press.

Heller, Monica. (2009). Doing Ethnography. Dans L. Wei \& M. G. Moyer (dir.), The Blackwell Guide to Research Methods in Bilingualism and Multilingualism (p. 249-262). Oxford, Victoria, MA : Blackwell.

Hymes, Dell Hymes. (1974). Foundations in Sociolinguistics: An Ethnographic Approach. University of Pennsylvania Press.

JASPERS, Jürgen. (2011). Strange Bedfellows: Appropriations of a Tainted Urban Dialect. Journal of Sociolinguistics, 15(4), 493-524.

Kerbrat-Orecchioni, Catherine. (2005). Le discours en interaction. Paris : Colin.

Kerbrat-Orecchioni, Catherine. (2006). Les actes de langage dans le discours. Paris : Colin.

Labov, William. (1972). Sociolinguistic Patterns. Oxford : Blackwell.

LAMBERT, Patricia. (2005). Les répertoires plurilectaux de jeunes filles d'un lycée professionnel. Une approche sociolinguistique éthnographique (Thèse de doctorat non publiée). Université Stendhal-Grenoble 3.

LAmbert, Patricia. (2009). (Dé)construction de clôtures identitaires dans un espace scolaire : un regard sociolinguistique impliqué. Lidil, 39, 43-56.

Mendoza-Denton, Norma. (2010). Individuals and Communities. Dans B. Johnstone, P. Kerswill \& R. Wodak (dir.), The SAGE Handbook of Sociolinguistics (p. 181-191). Los Angeles, Londres, New Dehli, Singapour, Washington D.C. : SAGE Publications.

Meyerhoff, Myriam. (2011). Introducing Sociolinguistics. Abingdon, Oxon; New York : Routledge.

Migge, Bettina \& Léglise, Isabelle. (2011). On the Emergence of New Language Varieties: The Case of the Eastern Maroon Creole in French Guiana. Dans L. Hinrichs \& J. T. Farquharson (dir.), Variation in the Caribbean (p. 181-199). Amsterdam : John Benjamins.

Migge, Bettina \& LÉGLISE, Isabelle. (2013). Exploring Language in a Multilingual Context: Variation, Interaction and Ideology in Language Documentation. Cambridge : Cambridge University Press.

Milroy, Lesley \& Gordon, Matthew. (2003). Sociolinguistics: Method and Interpretation. Malden, MA : Blackwell. 
MondadA, Lorenza. (2006). La question du contexte en ethnométhodologie et en analyse conversationnelle. Verbum, XXVIII(2-3), 111-151.

Myers-Scotton, Carol \& Bolonyai, Anne. (2001). Calculating Speakers: Codeswitching in a Rational Choice Model. Language in Society, 30(1), $1-28$.

Podesva, Robert. (2008). Three Sources of Stylistic Meaning. Texas Linguistic Forum (Proceedings of the symposium about language and society, Austin), 15(51), 1-10.

Schilling-Estes, Nathalie. (2002). Investigating Stylistic Variation. Dans J. K. Chambers, P. Trudgill \& N. Schilling-Estes (dir.), The Handbook of Language Variation and Change (4ééd., p. 375-401). Oxford, Victoria, MA : Blackwell Publishers.

Sedikides, Constantine \& Strube, Michael J. (1997). Self-Evaluation: To Thine Own Self Be Good, to Thine Own Self Be Sure, to Thine Own Self Be True, and to Thine Own Self Be Better. Dans M.P. Zanna (dir.), Advances in Experimental Social Psychology (vol. 29, p. 209-269). New York : Academic Press.

Snell, Julia. (2010). From Sociolinguistic Variation to Socially Strategic Stylisation. Journal of Sociolinguistics, 14(5), 630-656.

Sobrero, Alberto A. \& Miglietta, Annarita (2003). Note sul «parlar giovane» in Salento. Dans G. Ardrizzo \& D. Gambarara (dir.), La comunicazione giovane. Rubbettino : Soveria Mannelli.

TAJFEL, Henri. (1978). Interindividual Behavior and Intergroup Behavior. Dans H. Tajfel (dir.), Differentiation between Studies in the Social Psychology of Intergroup Relations (p. 27-60). Londres-New York : Academic Press.

Ursini, Flavia. (2005). La lingua dei giovani e i nuovi media: gli sms. Dans F. Fusco \& C. Marcato (dir.), Forme della comunicazione giovanile (p. 323-336). Rome : Il Calamo.

WaLd, Paul. (1990). Catégories de locuteur et catégories de langue dans l'usage du français en Afrique. Langage et société, 52, 5-21. 